\title{
Analyzing cost-effectiveness of ulnar and median nerve transfers to regain forearm flexion
}

\author{
*Arvin R. Wali, BA, ${ }^{1}$ Charlie C. Park, BS, ${ }^{2}$ Justin M. Brown, MD, ${ }^{1}$ and Ross Mandeville, MD1 \\ Departments of ${ }^{1}$ Neurological Surgery and ${ }^{2}$ Radiology, University of California, San Diego, California
}

OBJECTIVE Peripheral nerve transfers to regain elbow flexion via the ulnar nerve (Oberlin nerve transfer) and median nerves are surgical options that benefit patients. Prior studies have assessed the comparative effectiveness of ulnar and median nerve transfers for upper trunk brachial plexus injury, yet no study has examined the cost-effectiveness of this surgery to improve quality-adjusted life years (QALYs). The authors present a cost-effectiveness model of the Oberlin nerve transfer and median nerve transfer to restore elbow flexion in the adult population with upper brachial plexus injury.

METHODS Using a Markov model, the authors simulated ulnar and median nerve transfers and conservative measures in terms of neurological recovery and improvements in quality of life $(\mathrm{QOL})$ for patients with upper brachial plexus injury. Transition probabilities were collected from previous studies that assessed the surgical efficacy of ulnar and median nerve transfers, complication rates associated with comparable surgical interventions, and the natural history of conservative measures. Incremental cost-effectiveness ratios (ICERs), defined as cost in dollars per QALY, were calculated. Incremental cost-effectiveness ratios less than \$50,000/QALY were considered cost-effective. One-way and 2-way sensitivity analyses were used to assess parameter uncertainty. Probabilistic sampling was used to assess ranges of outcomes across 100,000 trials.

RESULTS The authors' base-case model demonstrated that ulnar and median nerve transfers, with an estimated cost of $\$ 5066.19$, improved effectiveness by 0.79 QALY over a lifetime compared with conservative management. Without modeling the indirect cost due to loss of income over lifetime associated with elbow function loss, surgical treatment had an ICER of $\$ 6453.41 /$ QALY gained. Factoring in the loss of income as indirect cost, surgical treatment had an ICER of $-\$ 96,755.42 /$ QALY gained, demonstrating an overall lifetime cost savings due to increased probability of returning to work. One-way sensitivity analysis demonstrated that the model was most sensitive to assumptions about cost of surgery, probability of good surgical outcome, and spontaneous recovery of neurological function with conservative treatment. Two-way sensitivity analysis demonstrated that surgical intervention was cost-effective with an ICER of $\$ 18,828.06 / Q A L Y$ even with the authors' most conservative parameters with surgical costs at $\$ 50,000$ and probability of success of $50 \%$ when considering the potential income recovered through returning to work. Probabilistic sampling demonstrated that surgical intervention was cost-effective in $76 \%$ of cases at a willingness-to-pay threshold of $\$ 50,000$ / QALY gained.

CONCLUSIONS The authors' model demonstrates that ulnar and median nerve transfers for upper brachial plexus injury improves QALY in a cost-effective manner.

https://thejns.org/doi/abs/10.3171/2016.12.FOCUS16469

KEY WORDS cost-effectiveness; nerve transfer; peripheral nerve surgery; upper brachial plexus injury; nerve transfer surgery; Oberlin procedure; median nerve transfer

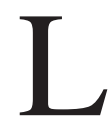
oss of elbow flexion secondary to upper brachial plexus injury is a complication associated with motor vehicle accidents and other forms of trauma, most commonly affecting males with a reported median age of 24-29 years. ${ }^{8,14,19}$ Loss of elbow flexion due to up- per brachial plexus injury leads to disability and dramatic reduction in quality of life (QOL) over a patient's remaining lifespan. ${ }^{11,20-22}$ Moreover, financial burdens on the individual and society are enormous. ${ }^{20-22}$ Treatment for brachial plexus injury includes both conservative manage-

ABBREVIATIONS CPT = Current Procedural Terminology; EMG = electromyography; ICER = incremental cost-effectiveness ratio; MRC = Medical Research Council; $\mathrm{QALY}=$ quality-adjusted life year; $\mathrm{QOL}=$ quality of life; WTP = willingness-to-pay.

SUBMITTED November 1, 2016. ACCEPTED December 7, 2016.

INCLUDE WHEN CITING DOI: 10.3171/2016.12.FOCUS16469.

* Mr. Wali and Mr. Park contributed equally to this work. 


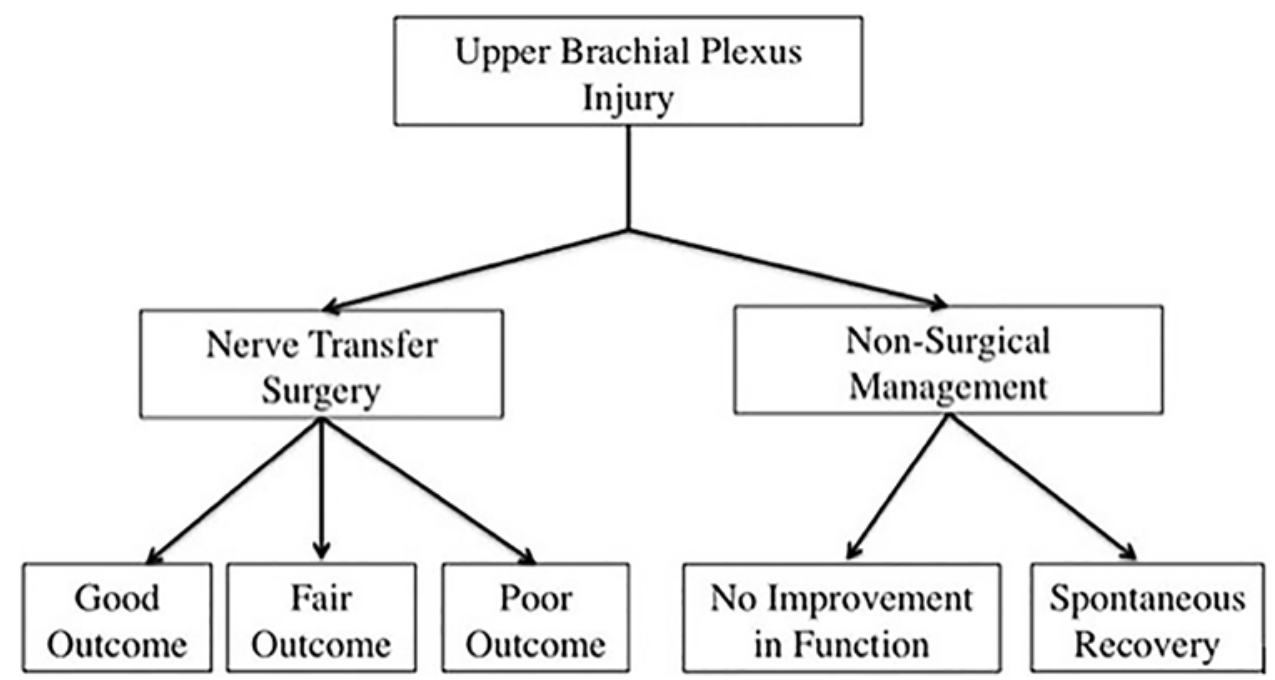

FIG. 1. Competing treatment strategies for upper brachial plexus injury after initial 4.5 months without spontaneous neurological recovery.

ment and operative management. Compared with conservative measures, surgical intervention via nerve transfers has been shown to significantly improve neurological function. $15,18,27$

Prompt surgical intervention within 6 months of injury has been associated with a more favorable outcome compared with delayed surgical treatment. ${ }^{4}$ For patients who undergo conservative treatment, $12 \%-41 \%$ may experience spontaneous recovery within the first 3 months. ${ }^{15,18}$ However, after this interval, spontaneous recovery and restoration of full neurological function becomes increasingly less likely. While multiple nerve transfers may be indicated to address additional deficits that result from an upper brachial plexus injury, ${ }^{26}$ restoration of elbow flexion remains the highest priority. ${ }^{3}$ Various nerve transfer approaches are available for restoring elbow flexion with remarkable rates of success ${ }^{3,7}$ and minimal reduction in functionality of the donor nerve. ${ }^{6}$ The Oberlin procedure, ${ }^{23}$ established in 1994, utilizes transfer of a fascicle of the ulnar nerve to the biceps branch of the musculocutaneous nerve to regain forearm flexion at the elbow. Similarly, a fascicle of the median nerve transferred to the biceps branch of the musculocutaneous nerve is also effective in improving elbow flexion. ${ }^{1,8,19}$ A double fascicular transfer that uses both ulnar and median nerve fascicles to reinnervate musculocutaneous branches to both biceps and brachialis muscles has been argued to provide additional strength. ${ }^{16}$ Although prior studies have investigated the comparative efficacy of these nerve transfer procedures, ${ }^{3}$ a cost-effectiveness study of surgical management compared with conservative management has not yet been conducted.

To our knowledge, we present the first cost-effectiveness model to demonstrate the incremental cost-effectiveness ratio (ICER) for ulnar and median nerve transfers in patients with upper brachial plexus injury. We aim to not only show cost-effectiveness of nerve transfer surgery on an individual and societal level, but also to highlight this underutilized time-sensitive surgical strategy and encourage early referral of potential surgical candidates by medical decision makers.

\section{Methods}

\section{Treatment Strategies}

Our decision model captures the choice between surgical and nonsurgical management 4.5 months after initial upper brachial plexus injury. We chose 4.5 months to capture the patient population with persistent neurological deficit with unlikely spontaneous recovery (Fig. 1). ${ }^{15,18}$ The competing treatment strategies in our models were as follows: Strategy 1, nonsurgical management of upper brachial plexus injury with a small chance of spontaneous neurological recovery; and Strategy 2, surgical management of upper brachial plexus injury via ulnar nerve transfer, median nerve transfer, or the combination of both branches of the ulnar and median nerves to reinnervate motor branches of the biceps to restore elbow flexion.

\section{Decision Model}

A decision-analysis model was constructed to assess the cost-effectiveness of ulnar and median nerve transfers in patients with loss of elbow flexion secondary to upper brachial plexus injury 4.5 months after injury. The role of additional nerve transfers to restore shoulder function was not included in this model. This model used carefully selected data from our literature review that included studies on health-related QOL and quality-adjusted life years (QALYs). The base scenario involved a 28 -year-old male patient, with 50 years of life expectancy, ${ }^{8}$ with loss of C-5 and C-6 function without spontaneous recovery 4.5 months after injury. Using a Markov model, each "patient" underwent 50 cycles ( 1 year per cycle) to model the lifetime QOL impact of each functional state. The decisionanalysis model is illustrated in Fig. 2.

\section{Outcomes}

Elbow flexion, categorized by the British Medical Research Council (MRC) score to assess neurological strength, was chosen as the primary clinical outcome. ${ }^{9,24,27}$ The 3 categories for surgical outcome were good, fair, and poor, each corresponding to MRC grades of $4-5,2-3$, and 


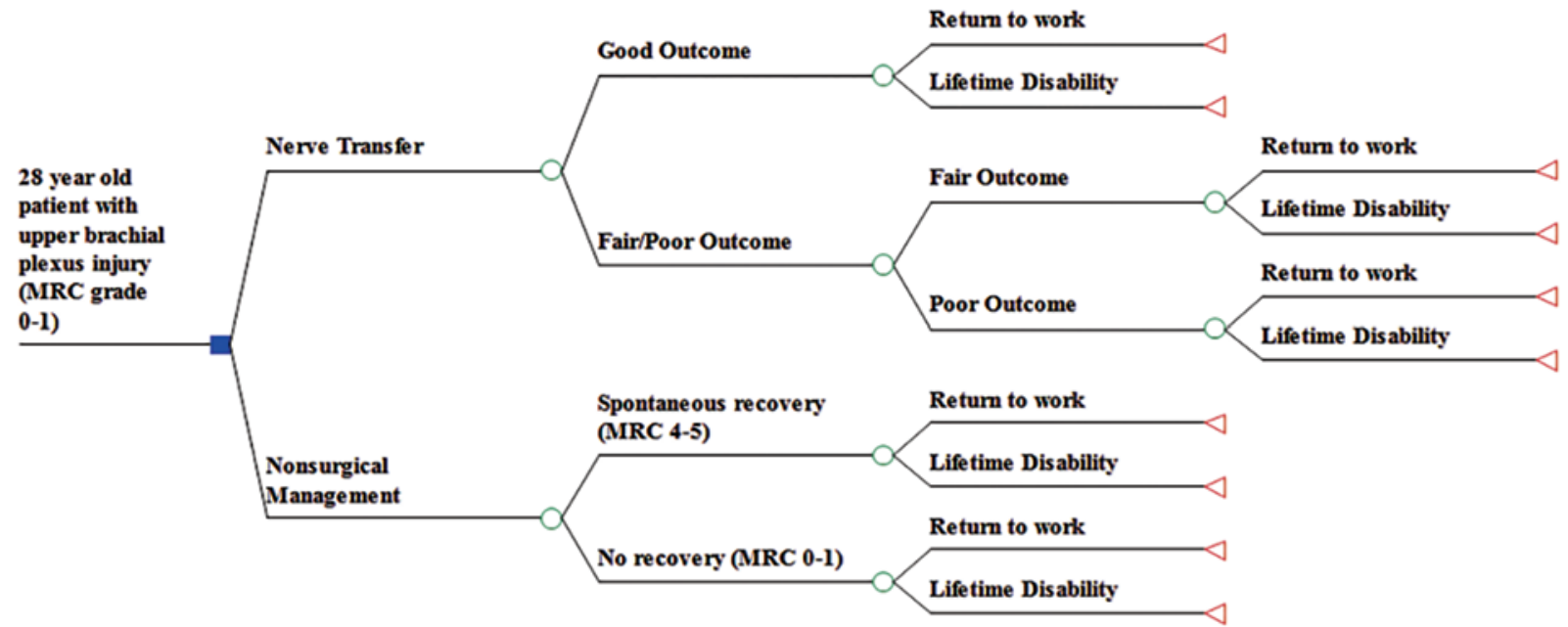

FIG. 2. Decision analysis model for musculoskeletal nerve injury with poor elbow flexion (MRC Grade $0-1$ ). The square decision node indicates the 2 options: to undergo surgical treatment or to undergo nonsurgical management. After undergoing nerve transfer surgery, the patient may either remain in a poor functional state or successfully achieve fair (MRC Grade 2-3) or good (MRC Grade 4-5) elbow flexion function. At each functional state, the patient may either return to work or remain disabled, resulting in loss of income.

$0-1$, respectively. The 2 outcomes associated with nonsurgical management were MRC Grade 4-5 with spontaneous recovery and MRC Grade 0-1 without neurological recovery. Our model grouped all patients with spontaneous recovery to MRC Grades $4-5$, a conservative assumption from the cost-effectiveness viewpoint. The corresponding QALYs of each MRC group, obtained from a previous study, were $0.89,0.82$, and 0.81 , respectively. ${ }^{2}$ Although QOL would be expected to improve between MRC Grades 2 and 3 with the capacity to perform movements against gravity, current literature associates approximately equivalent QALYs to these MRC grades. Our model assumed that equivalent restoration of elbow flexion to a given MRC grade whether by surgical intervention or by spontaneous recovery with nonsurgical management would result in equivalent QALY. Our model also assumed that improving from MRC Grades 0-1 to 4-5 regardless of surgical intervention or spontaneous recovery would result in equivalent likelihood to return to work.

\section{Model Probabilities}

Our model assumed that all patients had equivalent deficits in unilateral elbow flexion before making the choice of surgical versus nonsurgical management for their brachial plexus injury. Prior studies that have assessed upper brachial plexus injuries with nonsurgical treatment have demonstrated a $12 \%-41 \%$ chance of recovery of elbow flexion within the first 3 months. ${ }^{15,18}$ If spontaneous neurological recovery was not observed within the first 4.5 months, nonsurgical recovery was modeled to be unlikely over a lifetime. Our model conservatively assumed that even though spontaneous recovery of neurological function did not occur within the first 4.5 months, patients would have a $25 \%$ chance to make this degree of recovery in the subsequent 1-year period, a conservative assumption from a cost-effectiveness perspective. According to prior published data on ulnar nerve transfers, the Oberlin nerve transfer demonstrates an approximately 80\% success rate, defined as the proportion of patients achieving MRC Grade 4 or higher..$^{16}$ In a case series of 6 patients who underwent a double fascicular nerve transfer, the success rate of achieving MRC Grade 4-5 was 100\%. ${ }^{16}$ Meanwhile, a prospective case series showed that patients who underwent median nerve transfer demonstrated a $73 \%$ success rate of achieving MRC Grade 4-5. ${ }^{8}$ Given the comparable rates of success between ulnar nerve transfer, median nerve transfer, and combined procedures, our model used the most conservative estimate (73\%) of achieving MRC Grade 4-5 after surgical intervention. For the purposes of our model, if good surgical outcome was not obtained, patients had a 90\% chance of achieving MRC Grade 2-3. In the remaining $10 \%$ of cases, patients did not improve in MRC grade following surgery; these cases were considered surgical failures/poor outcomes.

Rates of surgical complications were derived from a prior study examining complications associated with comparable peripheral nerve surgeries involving the upper extremity. ${ }^{25}$ Our model included 2 potential complications of surgery: scar tenderness and wound infection. Our model assumed that these 2 complications were considered independent events. The annual probability of death due to natural causes at any given age was obtained from the US Census Bureau Data (https://www.cdc.gov/ nchs/nvss/mortality_methods.htm). All data on the probabilities of transition between functional states and the associated QALYs were collected retrospectively from the literature (Table 1).

\section{Costs}

All analyses were performed using a third-party payer's perspective. Total costs associated with surgery were determined by 2015 Medicare Fee-For-Service payment values (https://www.cms.gov/apps/physician-fee-schedule) using Current Procedural Terminology (CPT) codes with a 2015 conversion factor of $\$ 35.80$. CPT codes associated with pedicle nerve transfer surgery, facility charges, an- 
TABLE 1. Parameters used in our cost-effectiveness model

\begin{tabular}{|c|c|c|c|c|}
\hline Parameter & $\begin{array}{c}\text { Base-Case } \\
\text { Value }\end{array}$ & $S D^{*}$ & $\begin{array}{c}\text { Distribution for } \\
\text { Probabilistic } \\
\text { Sensitivity Analysis } †\end{array}$ & Authors \& Year \\
\hline Initial age (yrs) & 28 & 5.6 & Binomial & Cho et al., 2014 \\
\hline Probability of good surgical outcome & 0.73 & 0.146 & Beta & Cho et al., 2014 \\
\hline If good outcome not obtained, probability of surgical failure & 0.1 & 0.02 & Beta & Cho et al., 2014; Nath et al., 2006 \\
\hline Probability spontaneous recovery & 0.25 & 0.05 & Beta & Nagano, 1998; Kim et al., 2003 \\
\hline Baseline probability of returning to work w/ MRC Grade 1-3 & 0.55 & 0.11 & Beta & Felici et al., 2014 \\
\hline Improved probability of returning to work w/ MRC 4-5 & 0.7 & 0.14 & Beta & Felici et al., 2014 \\
\hline Cost of surgery & $\$ 5,066.19$ & $\$ 1,013.24$ & Gamma & $\begin{array}{l}\text { Centers for Medicare \& Medicaid Ser- } \\
\text { vices, } 2016 \text { (see text) }\end{array}$ \\
\hline Mean annual wage & $\$ 48,320$ & $\$ 9,664$ & Gamma & Bureau of Labor Statistics, 2015 (see text) \\
\hline Probability of wound infection & 0.09 & 0.018 & Beta & Song et al., 2012 \\
\hline Probability of scar tenderness & 0.09 & 0.018 & Beta & Song et al., 2012 \\
\hline MRC Grade 4-5 QALY & 0.89 & 0.11 & Beta & Ali et al., 2014 \\
\hline MRC Grade 2-3 QALY & 0.82 & 0.11 & Beta & Ali et al., 2014 \\
\hline MRC Grade 0-1 QALY & 0.81 & 0.14 & Beta & Ali et al., 2014 \\
\hline Scar tenderness QALY multiplier & 0.97 & 0.09 & Beta & Song et al., 2012 \\
\hline Wound infection QALY multiplier & 0.92 & 0.03 & Beta & Song et al., 2012 \\
\hline
\end{tabular}

* SD derived from literature when applicable; otherwise, $20 \%$ of the mean was used to provide SD if the value was not present in the literature.

$\dagger$ Binomial, beta, and gamma distributions refer to probabilistic distributions used in probabilistic sensitivity analysis.

esthesia, clinic visit costs, preoperative electromyography (EMG), preoperative cervical spine MRI, and 8 postoperative rehabilitation visits were summed to provide a total cost of $\$ 5066.19$ with surgical treatment. Components of surgical cost are listed in Table 2.

Brachial plexus injuries and corresponding disabilities contribute substantially to additional costs associated with loss of income and daily living. ${ }^{11,17}$ In 2014, Felici et al. demonstrated that patients with brachial plexus injury had a 45\% disability rate. After surgery and achieving MRC Grade 4 or 5 elbow flexion, the disability rate decreased to $30 \% .^{12}$ Using these data, we modeled our probability of return to work at $55 \%$ for those with MRC Grade 0-1 and 2-3, and at $70 \%$ for patients with MRC Grade 4-5. For those returning to work, we assumed a mean annual income of $\$ 48,320$ based on the Bureau of Labor Statistics 2015 data (https://www.bls.gov/oes/current/oes_nat.htm).

\section{Analysis}

All analyses of the model were performed using TreeAge Pro 2016 (TreeAge Software). Variables were tabulated into our model, and the incremental ICER was assessed. The model discounts utilities 3\% annually to adjust for future changes in QOL. ${ }^{13}$ The cost-effectiveness of surgical management compared with nonsurgical management was measured with an ICER, which is equivalent to the cost associated with each additional QALY gained. Results were considered cost-effective if the ICER was less than a willingness-to-pay (WTP) cutoff of $\$ 50,000 /$ QALY, a convention used in numerous cost-effectiveness studies. $^{5}$

Base-case analysis was performed to assess the cost-effectiveness of the change in QALY with surgical intervention compared with conservative management, with and without modeling the indirect cost associated with loss of income due to impaired elbow function.

One-way sensitivity analyses were performed to assess the cost-effectiveness of surgery irrespective of potential income earned over lifetime, with respect to changes in cost of surgery, probability of surgical success, probability of spontaneous recovery with conservative treatment, patient age, probability of surgical wound infection, and probability of surgical scar tenderness. Two-way sensitivity analysis was also performed to demonstrate costeffectiveness across ranges of probability of good surgical outcome and cost of surgery. All sensitivity analyses were performed without modeling potential lifetime income earned due to improvement in elbow function to ob-

\section{TABLE 2. Components of surgical costs}

\begin{tabular}{lrc}
\hline \multicolumn{1}{c}{ Direct Cost Component } & \multicolumn{1}{c}{ Fees $^{*}$} & CPT Code \\
\hline Surgery + facility fee & $\$ 2,240.89$ & 64905 \\
\hline Anesthesia & $\$ 670.00$ & 01710 \\
\hline New neurosurgery clinic visit & $\$ 207.29$ & 99205 \\
\hline Pre- \& postop surgical visits & $\$ 976.32$ & 99203 \\
\hline EMG conduction study & $\$ 197.62$ & 95910 \\
\hline Preop EMG & $\$ 92.00$ & 95886 \\
\hline Preop cervical spine MRI & $\$ 225.57$ & 72141 \\
\hline Rehabilitation & & \\
\hline New visit & $\$ 85.57$ & 97003 \\
\hline Follow-up & $\$ 370.93$ & 97004 \\
\hline Total cost & $\$ 5,066.19$ & \\
\hline
\end{tabular}

${ }^{*}$ Fees were derived from the 2015 Medicare Fee-For-Service payment values (https://www.cms.gov/apps/physician-fee-schedule). 
tain conservative estimates of the cost-effectiveness and benefits of surgery. Monte Carlo probabilistic sensitivity analysis was conducted with 100,000 iterations. ${ }^{10}$ Age distributions were modeled as binary distributions. Costs were modeled by gamma distributions to capture a range of costs between zero and infinity. Probabilities and health utilities defined by QOL were modeled after beta distributions between 0 and 1 . When possible, standard deviations associated with given values were obtained from the literature. When standard deviations were not available, a $20 \%$ standard deviation was assigned to the mean. Given the high variance associated with QALYs and MRC grade, Monte Carlo probabilistic sensitivity analysis was performed with and without taking into account the differences due to variance among MRC grades.

\section{Results \\ Base Case Analysis}

Our model demonstrated that surgical intervention is strongly effective, yielding 21.23 QALYs in the surgical group compared with 20.44 QALYs with nonsurgical management. Our cost-effectiveness analysis showed that surgical management was cost-effective with an ICER of \$6453.41/QALY when not including potential income recovered through returning to work with MRC Grade 4-5 elbow function. When considering potential lifetime income gained with improved elbow function at MRC Grade 4-5 elbow function, surgical management was cost-effective with an ICER of $-\$ 96,755.42$. This negative ICER indicates that, despite costs associated with surgery, the potential income recovered through returning to work may result in monetary gain.

\section{One-Way Sensitivity Analysis}

Cost-effectiveness was most sensitive to changes in cost of surgery, probability of good surgical outcome, and probability of spontaneous recovery. Changes in cost of surgery from $\$ 5000.00$ to $\$ 50,000$ yielded ICERs ranging from $\$ 6369.10$ to $\$ 63,690.98 / \mathrm{Q} A L Y$. Regardless of the potential income earned over lifetime due to a successful functional outcome, ulnar and median nerve transfer demonstrated cost-effectiveness up to a threshold cost of $\$ 39,252.03$. Changes in probability of good surgical outcome (MRC Grade 4-5) from 50\% to $100 \%$ yielded ICERs between $\$ 4008.00$ to $\$ 13,437.40 /$ QALY gained, demonstrating cost-effectiveness irrespective of the income gained through returning to work at a willingness to pay of $\$ 50,000 / \mathrm{QALY}$ gained. Chances of spontaneous recovery in the nonsurgical group in the year following the initial 4.5 months of injury without spontaneous recovery were modeled between $0 \%$ and $60 \%$, resulting in ICERs that ranged from $\$ 4026.68$ to $\$ 41,295.72$, again demonstrating cost-effectiveness (Table 3).

\section{Two-Way Sensitivity Analysis}

Two-way sensitivity analysis (Fig. 3) illustrated the most cost-effective strategy, with surgical costs ranging from $\$ 5066.19$ to $\$ 50,000$ and probability of good surgical outcome ranging from $50 \%$ to $100 \%$. For the surgical treatment to be the more cost-effective strategy
TABLE 3. One-way sensitivity analysis demonstrating changes in ICER

\begin{tabular}{ccc}
\hline Parameter & $\begin{array}{c}\text { Value Used } \\
\text { for Sensitivity } \\
\text { Analysis }\end{array}$ & $\begin{array}{c}\text { ICER (\$/QALY gained), } \\
\text { Disregarding Income Loss } \\
\text { w/ Disability }\end{array}$ \\
\hline Cost of surgery* $^{*}$ & $\$ 5,000.00 \dagger$ & $\$ 6,369.10$ \\
& $\$ 16,250.00$ & $\$ 20,699.57$ \\
& $\$ 27,500.00$ & $\$ 35,030.04$ \\
& $\$ 38,750.00$ & $\$ 49,360.51$ \\
Probability of good & $50,000.00$ & $\$ 63,690.98$ \\
surgical outcome & $60 \%$ & $\$ 13,437.40$ \\
& $70 \% \ddagger$ & $\$ 9,137.80$ \\
& $80 \% \$$ & $\$ 6,922.72$ \\
taneous recovery & $90 \% \uparrow$ & $\$ 5,572.02$ \\
after 4.5 mos & $100 \% * *$ & $\$ 4,662.34$ \\
w/o neurological & $10 \%$ & $\$ 4,008.00$ \\
improvement & $20 \%$ & $\$ 4,026.68$ \\
& $30 \%$ & $\$ 4,739.59$ \\
& $40 \%$ & $\$ 5,759.24$ \\
\hline Probability of spon- & $\$ 7,337.86$ \\
& $50 \%$ & $\$ 10,108.68$ \\
\hline
\end{tabular}

* Nerve transfer surgery remained cost-effective across the ranges of surgical cost (threshold of cost-effectiveness: $\$ 39,252.03$ ), probability of good surgical outcome, and probability of spontaneous recovery.

$\dagger$ The approximate cost of a nerve transfer procedure in this study was $\$ 5,066.19$.

$\ddagger$ Cho et al., 2010; approximate success rate of median nerve transfer (73\%) based on retrospective data.

$\S$ Mackinnon et al., 2005; approximate success rate of Oberlin procedure based on a meta-analysis.

I Nath et al., 2006; approximate success rate of median nerve transfer.

${ }^{* *}$ Mackinnon et al., 2005; approximate success rate of double fascicular transfer based on a meta-analysis.

at the WTP cutoff, the cost of surgery must not exceed $\$ 18,851.08$ where success of achieving MRC Grade $4-5$ is $50 \%$. Moreover, surgical intervention can remain cost-effective for up to $\$ 50,000$ when surgical success rate is $85 \%$ or greater. When accounting for possible income gained with improved elbow function, nerve transfer surgery was found to be cost saving, yielding an ICER within a WTP less than \$50,000/QALY gained across the entire range of probability of good outcome from $50 \%$ to $100 \%$ and surgical cost ranging from $\$ 5066.19$ to $\$ 50,000$. Even our most conservative parameters in which probability of surgical success was $50 \%$ and cost of surgery was $\$ 50,000$ yielded an ICER of $\$ 18,828.06 /$ QALY, demonstrating cost-effectiveness.

\section{Probabilistic Sensitivity Analysis}

Monte Carlo probabilistic sensitivity analysis of 100,000 iterations, with surgical success and spontaneous recovery probabilities, QALYs, potential income lost due to disability, and cost of surgery modeled as distributions to account for variations, showed that surgical intervention is more cost-effective than nonsurgical management in $76 \%$ of cases at the WTP cutoff (Fig. 4). 


\section{Discussion}

Quantification of outcomes is a cornerstone of evidencebased medicine. Surgical interventions are increasingly scrutinized, and outcome measurements are constantly being refined. The ultimate outcome of interest for patients is QOL, and, in our progressively cost-conscious health care system/society, it is imperative to identify treatments that are cost-effective in improving QOL.

In previously published studies, median nerve transfer, ulnar nerve transfer, and a combination of ulnar and median nerve transfer in patients with upper brachial plexus injury have been demonstrated to be effective surgical strategies for restoring elbow flexion. ${ }^{16,19}$ We present the first study, to our knowledge, to assess the cost-effectiveness of the procedures. Despite the small chance of spontaneous recovery in upper brachial plexus injuries after 4.5 months of injury, ${ }^{18}$ our model demonstrated that surgical intervention is highly cost-effective compared with conservative management for improving the QALYs for patients with upper brachial plexus injury while also increasing the likelihood that a patient may return to work and earn income over lifetime. Both the increase in income and the improvements in QALY across a lifetime through surgical intervention made nerve transfer surgery cost-effective at a WTP of less than $\$ 50,000 /$ QALY even at the most conservative estimates of a $50 \%$ chance of surgical success and $\$ 50,000$ cost of surgery.

Our study demonstrated a negative ICER (-\$96,755.42/ QALY) when modeling the income lost due to elbow flexion disability and the increased probability of a patient returning to work after a good surgical outcome. This highlights that the magnitude of potential income gained through improving elbow function exceeds the costs of nerve transfer surgery. Importantly, the result of this study demonstrates the cost-effectiveness of the Oberlin nerve transfer and comparable procedures utilizing the median nerve, ulnar nerve, or a combination of both. ${ }^{8,16,19}$

There are several limitations to our model. This model is based on assumptions and values obtained from prior studies, many of which are retrospective single-center studies that have not been externally validated in prospective, randomized trial settings. The costs of surgery used in this study were derived from Medicare values and may not capture costs across different health care settings. Probability of success may vary from surgeon to surgeon and certain facilities may not offer nerve transfer surgery. Our model does not incorporate additional costs associated with surgical complications, other types of surgical complications, or other ancillary surgical costs including cost of pain medications and antibiotics. However, the range of surgical costs in our 1-way and 2-way sensitivity analyses accounted for additional costs that were not included and demonstrated that surgical intervention is cost-effective up to $\$ 18,851.08$ at a good surgical outcome rate of only $50 \%$ and cost-effective up to $\$ 50,000$ for surgical costs with a good surgical outcome rate of $85 \%$. When considering the potential income recovered through returning to work, surgical intervention was cost-effective across the entire range of surgical cost from $\$ 5066.19$ to $\$ 50,000$ and the entire range of probability of good outcome from $50 \%$ to $100 \%$.

Our model does not take into account possible neuro-

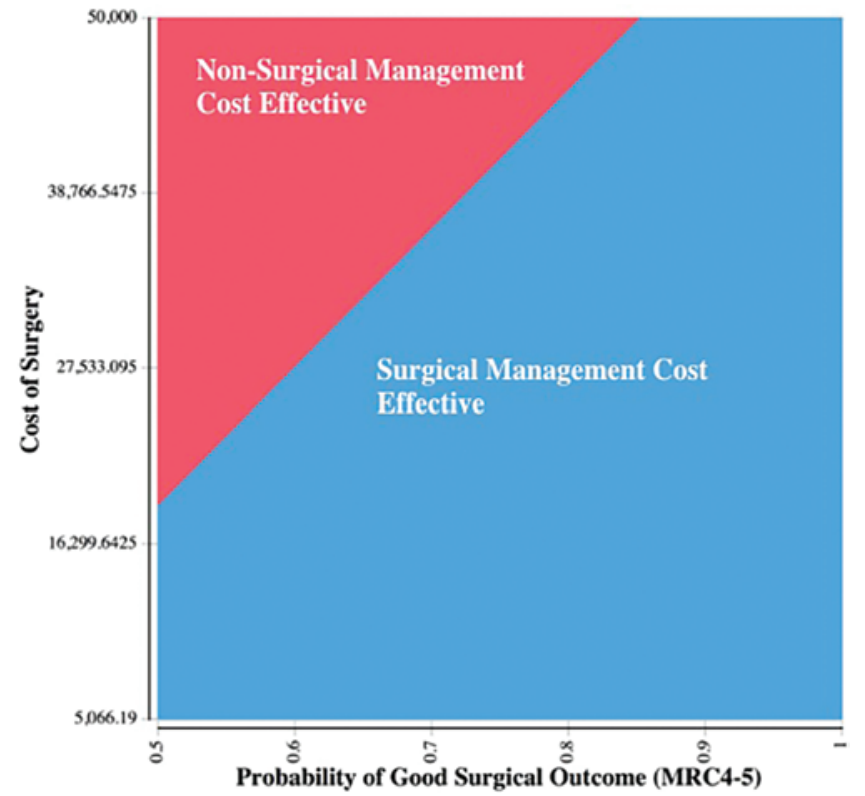

FIG. 3. Two-way sensitivity analysis for the base-case scenario of nerve transfer procedures in brachial plexus injury. The x-axis illustrates the range of probability of good outcome (MRC Grade 4-5), while the $y$-axis illustrates the range of cost of surgery from $\$ 5066.19$ to $\$ 50,000$. The red and blue areas indicate where nonsurgical management or surgical treatment, respectively, is the most cost-effective strategy. The boundary line between the red and blue areas represents the WTP cutoff of $\$ 50,000 / Q A L Y$, and theoretically represents where nonsurgical management and surgical treatment are both equally cost-effective. This 2-way sensitivity analysis did not account for the indirect cost due to loss of income over lifetime due to impaired elbow function. When accounting for income gained with improved elbow function, nerve transfer surgery is always cost-effective across the range of probability of good outcome and surgery cost represented in this plot.

logical injury as a complication associated with surgery. Oberlin in 1996 reported postoperative weakness after nerve transfer surgery, but the weakness was not permanent. ${ }^{23}$ Moreover, the decrease in QALY associated with functional loss due to fascicular injury of the median or ulnar nerve is not yet established within the literature, limiting our cost-effectiveness model from analyzing surgical complications that may decrease function. Another limitation was the large variance in QALYs at a given MRC grade, ${ }^{2}$ which could suggest potential scenarios in which patients with MRC Grade 4-5 functionality have a lower QALY than patients with an MRC Grade 0-1. Large variance in QALYs across MRC grades highlights the importance and need for more preference-based QOL research to lay the foundation of more precise research in medical decision-making. Despite this large variance, surgical intervention remained the more cost-effective choice in $76 \%$ of iterations through Monte Carlo probabilistic simulation, highlighting the utility of nerve transfer surgery over conservative management alone. Future studies are needed to identify and validate precise QALY values associated with different MRC grades of elbow flexion function.

Despite these limitations, this study provides an important perspective for the role of the median nerve transfer, ulnar nerve transfer, or a combination of both for upper 


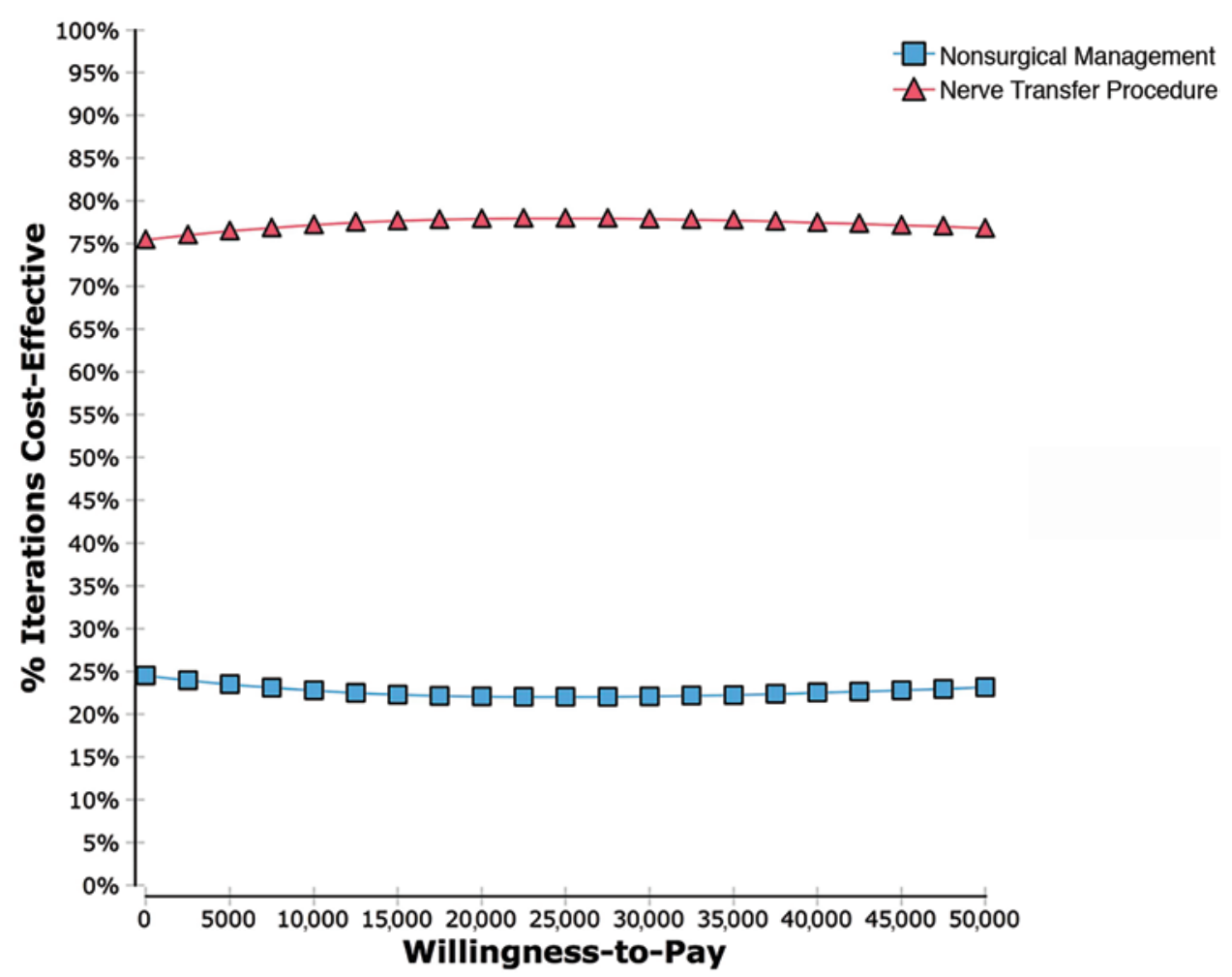

FIG. 4. Cost-effectiveness acceptability curve demonstrating that as WTP increases, surgical management continues to dominate as the cost-effective strategy for $76 \%$ of probabilistic iterations.

brachial plexus injury. In the absence of spontaneous neurological recovery within 4.5 months of the initial nerve injury, these surgical procedures are cost-effective compared with nonsurgical management to restore elbow flexion after upper brachial plexus injury. These findings should be considered when recommending potential surgical candidates to receive nerve transfer surgery.

\section{Conclusions}

Upper brachial plexus injury can result in loss of elbow flexion, leading to disability and reductions in QOL. Our study demonstrates that Oberlin nerve transfer and similar procedures are highly cost-effective strategies for restoring elbow flexion function and improving QOL for patients with upper brachial plexus injury.

\section{Acknowledgments}

A.R.W. and C.C.P. are funded through the NIH TL1 predoctoral grant (grant nos. 1TL1TR001443 and TL1TR00098, respectively).

\section{References}

1. Al-Qattan MM, Al-Kharfy TM: Median nerve to biceps nerve transfer to restore elbow flexion in obstetric brachial plexus palsy. BioMed Res Int 2014:854084, 2014

2. Ali ZS, Bakar D, Li YR, Judd A, Patel H, Zager EL, et al: Utility of delayed surgical repair of neonatal brachial plexus palsy. J Neurosurg Pediatr 13:462-470, 2014

3. Ali ZS, Heuer GG, Faught RW, Kaneriya SH, Sheikh UA, Syed IS, et al: Upper brachial plexus injury in adults: com- parative effectiveness of different repair techniques. J Neurosurg 122:195-201, 2015

4. Bentolila V, Nizard R, Bizot P, Sedel L: Complete traumatic brachial plexus palsy. Treatment and outcome after repair. J Bone Joint Surg Am 81:20-28, 1999

5. Braithwaite RS, Meltzer DO, King JT Jr, Leslie D, Roberts MS: What does the value of modern medicine say about the $\$ 50,000$ per quality-adjusted life-year decision rule? Med Care 46:349-356, 2008

6. Brown JM, Shah MN, Mackinnon SE: Distal nerve transfers: a biology-based rationale. Neurosurg Focus 26(2):E12, 2009

7. Brown JM, Vivio N, Sheean GL: The clinical practice of reconstructive neurosurgery. Clin Neurol Neurosurg 114:506-514, 2012

8. Cho AB, Paulos RG, de Resende MR, Kiyohara LY, Sorrenti L, Wei TH, et al: Median nerve fascicle transfer versus ulnar nerve fascicle transfer to the biceps motor branch in C5-C6 and C5-C7 brachial plexus injuries: nonrandomized prospective study of 23 consecutive patients. Microsurgery 34:511-515, 2014

9. Compston A: Aids to the investigation of peripheral nerve injuries. Medical Research Council: Nerve Injuries Research Committee. His Majesty's Stationery Office: 1942; pp. 48 (iii) and 74 figures and 7 diagrams; with aids to the examination of the peripheral nervous system. By Michael O'Brien for the Guarantors of Brain. Saunders Elsevier: 2010; pp. [8] 64 and 94 Figures. Brain 133:2838-2844, 2010

10. Concato J, Feinstein AR: Monte Carlo methods in clinical research: applications in multivariable analysis. J Investig Med 45:394-400, 1997

11. Cross JD, Ficke JR, Hsu JR, Masini BD, Wenke JC: Battlefield orthopaedic injuries cause the majority of long-term disabilities. J Am Acad Orthop Surg 19 (Suppl 1):S1-S7, 2011 
12. Felici N, Zaami S, Ciancolini G, Marinelli E, Tagliente D, Cannatà C: Cost analysis of brachial plexus injuries: variability of compensation by insurance companies before and after surgery. Handchir Mikrochir Plast Chir 46:85-89, 2014

13. Hunink MG: In search of tools to aid logical thinking and communicating about medical decision making. Med Decis Making 21:267-277, 2001

14. Kaiser R, Waldauf P, Haninec P: Types and severity of operated supraclavicular brachial plexus injuries caused by traffic accidents. Acta Neurochir (Wien) 154:1293-1297, 2012

15. Kim DH, Cho YJ, Tiel RL, Kline DG: Outcomes of surgery in 1019 brachial plexus lesions treated at Louisiana State University Health Sciences Center. J Neurosurg 98:1005-1016, 2003

16. Mackinnon SE, Novak CB, Myckatyn TM, Tung TH: Results of reinnervation of the biceps and brachialis muscles with a double fascicular transfer for elbow flexion. J Hand Surg Am 30:978-985, 2005

17. Masini BD, Waterman SM, Wenke JC, Owens BD, Hsu JR, Ficke JR: Resource utilization and disability outcome assessment of combat casualties from Operation Iraqi Freedom and Operation Enduring Freedom. J Orthop Trauma 23:261266, 2009

18. Nagano A: Treatment of brachial plexus injury. J Orthop Sci 3:71-80, 1998

19. Nath RK, Lyons AB, Bietz G: Physiological and clinical advantages of median nerve fascicle transfer to the musculocutaneous nerve following brachial plexus root avulsion injury. J Neurosurg 105:830-834, 2006

20. Novak CB, Anastakis DJ, Beaton DE, Katz J: Patient-reported outcome after peripheral nerve injury. J Hand Surg Am 34:281-287, 2009

21. Novak CB, Anastakis DJ, Beaton DE, Mackinnon SE, Katz $\mathrm{J}$ : Biomedical and psychosocial factors associated with disability after peripheral nerve injury. J Bone Joint Surg Am 93:929-936, 2011

22. Novak CB, Anastakis DJ, Beaton DE, Mackinnon SE, Katz J: Validity of the Patient Specific Functional Scale in patients following upper extremity nerve injury. Hand (NY) 8:132138,2013
23. Oberlin C, Béal D, Leechavengvongs S, Salon A, Dauge MC, Sarcy JJ: Nerve transfer to biceps muscle using a part of ulnar nerve for C5-C6 avulsion of the brachial plexus: anatomical study and report of four cases. J Hand Surg Am 19:232-237, 1994

24. Ray WZ, Mackinnon SE: Clinical outcomes following median to radial nerve transfers. J Hand Surg Am 36:201-208, 2011

25. Song JW, Chung KC, Prosser LA: Treatment of ulnar neuropathy at the elbow: cost-utility analysis. J Hand Surg Am 37:1617-1629, 1629.e1-1629.e3, 2012

26. Tsai YJ, Su FC, Hsiao CK, Tu YK: Comparison of objective muscle strength in C5-C6 and C5-C7 brachial plexus injury patients after double nerve transfer. Microsurgery 35:107114, 2015

27. van der Ploeg RJ, Oosterhuis HJ, Reuvekamp J: Measuring muscle strength. J Neurol 231:200-203, 1984

\section{Disclosures}

The authors report no conflict of interest concerning the materials or methods used in this study or the findings specified in this paper.

\section{Author Contributions}

Conception and design: all authors. Acquisition of data: Mandeville, Wali, Park. Analysis and interpretation of data: Mandeville, Wali, Park. Drafting the article: Mandeville, Wali, Park. Critically revising the article: all authors. Reviewed submitted version of manuscript: all authors. Approved the final version of the manuscript on behalf of all authors: Mandeville. Statistical analysis: Mandeville, Wali, Park. Administrative/technical/material support: Mandeville.

\section{Correspondence}

Ross Mandeville, Department of Neurological Surgery, University of California, San Diego, MC 8893, 200 West Arbor Dr., San Diego, CA 92103.email: rmandeville@ucsd.edu. 